\title{
Entropy approach for 2D velocity distribution in open-channel flow
}

\section{Gustavo Marini , Giuseppe De Martino , Nicola Fontana , Mauro Fiorentino \& Vijay P. Singh}

To cite this article: Gustavo Marini , Giuseppe De Martino , Nicola Fontana , Mauro Fiorentino \& Vijay P. Singh (2011) Entropy approach for 2D velocity distribution in open-channel flow, Journal of Hydraulic Research, 49:6, 784-790, DOI: 10.1080/00221686.2011.635889

To link to this article: http://dx.doi.org/10.1080/00221686.2011.635889

\section{Published online: 21 Nov 2011.}

\section{Submit your article to this journal 저}

Џlll Article views: 404

Q View related articles $\sqsubset$

Citing articles: 25 View citing articles 4 
Research paper

\title{
Entropy approach for 2D velocity distribution in open-channel flow
}

\author{
GUSTAVO MARINI, Assistant Professor, Dipartimento di Ingegneria, University of Sannio, Piazza Roma 21, Benevento \\ 82100, Italy. \\ Email: gustavo.marini@unisannio.it (author for correspondence)
}

GIUSEPPE DE MARTINO, Professor, Dipartimento di Ingegneria Idraulica, Geotecnica e Ambientale, University of Napoli

"Federico II", via Claudio 21, Napoli 80125, Italy.

Email: giudemar@unina.it

NICOLA FONTANA, Associate Professor, Dipartimento di Ingegneria, University of Sannio, Piazza Roma 21, Benevento 82100 , Italy.

Email:fontana@unisannio.it

MAURO FIORENTINO, Professor, Dipartimento di Ingegneria e Fisica dell'Ambiente, University of Basilicata, Viale dell'Ateneo 10, Potenza 85100, Italy.

Email: mauro.fiorentino@unibas.it

VIJAY P. SINGH, Professor, Department of Biological and Agricultural Engineering, Texas A\&M University, 2117 TAMU, College Station, TX 77842-2117, USA; Department of Civil and Environmental Engineering, Texas A\&M University, 2117 TAMU, College Station, TX 77842-2117, USA.

Email:vsingh@tamu.edu

\begin{abstract}
In this paper, considering time-averaged velocity as a random variable, two-dimensional (2D) velocity distributions in open-channel flow have been derived based on the Shannon entropy concept and the principle of maximum entropy. The velocity distributions so derived have limited practical use, since they contain too many parameters that need to be experimentally calibrated and hence are not convenient to apply. This work develops a new entropy-based approach for deriving a 2D velocity distribution in open-channel flow, thereby investigating a rectangular geometric domain. The derived distribution is parsimonious, and the values determined using the proposed distribution are found to be in good agreement with the experimentally-measured velocity values.
\end{abstract}

Keywords: Entropy, flow measurement, open-channel flow, Shannon entropy, streamflow, velocity distribution

\section{Introduction}

The velocity distribution is fundamental in the hydraulic modelling of natural rivers, including modelling of sediment and contaminant transport, the design of channels, river training works, and hydraulic structures, or the development of rating curves. For an accurate velocity distribution over the full range of flow stages, velocity measurements must be carried out during flood events, which result in considerable difficulties and danger. Therefore, it is desirable to define in a simple and quick way the velocity distribution based only a few sampling points.
Considering that the velocity is subjected to uncertainties arising from natural and/or man-made causes, Chiu (1987) considered time-averaged velocity as a probabilistic variable, unlike traditional approaches which assume it to be a deterministic variable (Tang and Knight 2009). Since then, one-dimensional (1D) velocity distributions have been derived and employed in hydraulics (Chiu 1987, 1989, Barbé et al. 1991, Chiu and Said 1995, Kirkgoz et al. 2009), whereas two-dimensional (2D) velocity distribution has resulted from the extension of the entropy-based method (Chiu 1988, Chiu and Hsu 2006). Although the latter distribution has

Revision received 3 October 2011/Open for discussion until 30 June 2012. 
been used in theoretical investigations, its practical use is limited.

Therefore, the objective of this study is to derive a $2 \mathrm{D}$ velocity distribution using the Shannon entropy, so that the coordinate system is mathematically sound and the distribution is parsimonious, and to interpret the distribution parameters in terms of hydraulic characteristics.

\section{Derivation of 2D velocity distribution using entropy}

\subsection{The principle of maximum entropy}

To obtain the least biased probability density function (PDF) of velocity, the entropy of the velocity distribution must be maximized subject to the specified constraints. The reason for entropy maximization stems from the principle of maximum entropy (POME) postulated by Jaynes (1957), stating that any system in the equilibrium state under steady constraints tends to maximize its entropy. As a river reaches a dynamic (or quasidynamic) equilibrium, the entropy must attain its maximum value (Singh et al. 2003). The POME states that the velocity distribution obtained by entropy maximization will be least biased towards what is not known about the velocity and most biased towards what is known via constraints. This principle is also consistent with the theory of minimum energy dissipation proposed by Yang $(1972,1976)$. The constraints have physical meaning as discussed below. Therefore, the derivation of the $2 \mathrm{D}$ velocity distribution involves the following steps: (1) definition of the continuous form of the Shannon entropy, (2) specification of velocity information in terms of constraints, (3) maximization of entropy and derivation of the probability distribution of velocity, (4) derivation of the $2 \mathrm{D}$ velocity distribution, (5) expression of the $2 \mathrm{D}$ probability distribution, and (6) estimation of parameters.

\subsection{The Shannon entropy}

Let temporally-averaged velocity $u$ be treated as a random variable with $f(u)$ as its PDF. Then, the Shannon (1948) entropy of $u$ or $f(u)$ is defined as

$$
H(u)=-\int f(u) \ln [f(u)] \mathrm{d} u
$$

in which $f(u) \mathrm{d} u$ represents the probability of $u$ between $u$ and $u+\mathrm{d} u$. Entropy $H(u)$ quantifies the uncertainty associated with $u$ or its PDF. Here, the objective is to derive $f(u)$ by applying the POME (Jaynes 1957), subject to specified constraints on velocity.

\subsection{Specification of constraints}

Information on velocity can be derived from the laws of conservation of mass, momentum, and energy. Chiu (1987) and later Barbé et al. (1991) observed that for purposes of deriving timeaveraged velocity distributions, it is sufficient to use only mass conservation. Therefore, constraints for the PDF of velocity $f(u)$ are expressed with $u_{\max }$ as the maximum value of $u$ at or below the water surface and $\bar{u}$ as the mean velocity as

$$
\int_{0}^{u_{\max }} f(u) \mathrm{d} u=1
$$

and

$$
\int_{0}^{u_{\max }} u f(u) \mathrm{d} u=\bar{u}
$$

The average velocity constraint in Eq. (3) is derived from, and hence satisfies, mass conservation (Barbé et al. 1991). This formulation does not include energy or momentum conservation.

\subsection{Maximization of entropy and probability distribution}

In accordance with the POME, $H(u)$ can be maximized subject to Eqs. (2) and (3) using the method of Lagrange multipliers and recalling the calculus of variation, resulting in

$$
f(u)=\exp \left(\lambda_{1}+\lambda_{2} u-1\right)
$$

where $\lambda_{1}$ and $\lambda_{2}$ are Lagrange multipliers.

\subsection{The $2 D$ velocity distribution}

Consider a 2D domain $(x, y)$, with $x$ as the transverse direction and $y$ as the vertical direction measured from the bed upward positive. Thus, $u=u(x, y)$ and its $\operatorname{PDF}$ is $f[u(x, y)]$ and the cumulative probability distribution function (CDF) is $F[u(x, y)]$. Taking the partial derivatives of $F(u)$ with respect to $x$ and $y$,

$$
\begin{aligned}
& \frac{\partial F(u)}{\partial x}=\frac{\mathrm{d} F(u)}{\mathrm{d} u} \frac{\partial u}{\partial x}=f(u) \frac{\partial u}{\partial x} \\
& \frac{\partial F(u)}{\partial y}=\frac{\mathrm{d} F(u)}{\mathrm{d} u} \frac{\partial u}{\partial y}=f(u) \frac{\partial u}{\partial y}
\end{aligned}
$$

From Eq. (4), Eqs. (5) are rewritten as

$$
\begin{aligned}
& \exp \left(\lambda_{2} u\right) \frac{\partial u}{\partial x}=\exp \left(1-\lambda_{1}\right) \frac{\partial F(u)}{\partial x} \\
& \exp \left(\lambda_{2} u\right) \frac{\partial u}{\partial y}=\exp \left(1-\lambda_{1}\right) \frac{\partial F(u)}{\partial y}
\end{aligned}
$$

Let quantity $\exp \left(\lambda_{2} u\right)$ be $w$ so that the partial derivatives of $w$ with respect to $x$ and $y$ are

$$
\begin{aligned}
& \frac{\partial w}{\partial x}=\frac{\partial \exp \left(\lambda_{2} u\right)}{\partial x}=\lambda_{2} \exp \left(\lambda_{2} u\right) \frac{\partial u}{\partial x} \\
& \frac{\partial w}{\partial y}=\frac{\partial \exp \left(\lambda_{2} u\right)}{\partial y}=\lambda_{2} \exp \left(\lambda_{2} u\right) \frac{\partial u}{\partial y}
\end{aligned}
$$


Substitution of Eqs. (7) into Eqs. (6) gives the following system of equations:

$$
\begin{aligned}
& \frac{\partial w}{\partial x}=\lambda_{2} \exp \left(1-\lambda_{1}\right) \frac{\partial F(u)}{\partial x} \\
& \frac{\partial w}{\partial y}=\lambda_{2} \exp \left(1-\lambda_{1}\right) \frac{\partial F(u)}{\partial y}
\end{aligned}
$$

Equations (8) can be integrated using the Leibniz rule that states

$$
\int_{0,0}^{(x, y)} \frac{\partial w}{\partial x} \mathrm{~d} x+\frac{\partial w}{\partial y} \mathrm{~d} y=w(x, y)-w(0,0)
$$

Because the point $(0,0)$ lies on solution domain's contour, $u$ at this point is equal to 0 , and the right-hand side of Eq. (9) becomes

$$
\begin{aligned}
w(x, y)-w(0,0) & =w(x, y)-\exp \left(\lambda_{2} u\right) \\
& =w(x, y)-\exp (0)=w(x, y)-1
\end{aligned}
$$

The definite integral of the first part of Eq. (9) is calculated at a generic point $(\bar{x}, \bar{y})$ identified by means of a polygonal curve that starts from $(0,0)$, passes across $(\bar{x}, 0)$, and ends at $(\bar{x}, \bar{y})$ so that

$$
\begin{gathered}
\int_{0,0}^{(\bar{x}, \bar{y})} \frac{\partial F(u)}{\partial y} \lambda_{2} \exp \left(1-\lambda_{1}\right) \mathrm{d} y+\frac{\partial F(u)}{\partial x} \lambda_{2} \exp \left(1-\lambda_{1}\right) \mathrm{d} x \\
=\int_{0}^{\bar{y}} \frac{\partial F(u)}{\partial y} \lambda_{2} \exp \left(1-\lambda_{1}\right) \mathrm{d} y=\lambda_{2} \exp \left(1-\lambda_{1}\right) F(u)
\end{gathered}
$$

in which $(\bar{x}, \bar{y})$ represents a point of the domain.

The right-hand side of Eq. (11) can be equated to the righthand side of Eq. (10) to obtain

$$
w(x, y)=1+\lambda_{2} \exp \left(1-\lambda_{1}\right) F(u)
$$

Because $w(x, y)=\exp \left(\lambda_{2} u\right)$, Eq. (12) is rewritten as

$$
\exp \left[\lambda_{2} u(x, y)\right]=1+\lambda_{2} \exp \left(1-\lambda_{1}\right) F(u(x, y))
$$

resulting for $u(x, y)$ in

$$
u(x, y)=\frac{1}{\lambda_{2}} \ln \left[1+\lambda_{2} \exp \left(1-\lambda_{1}\right) F(u(x, y))\right]
$$

Equation (14) contains the two Lagrange multipliers $\lambda_{1}$ and $\lambda_{2}$, which are determined using Eqs. (2) and (3). Integration of Eq. (2) yields

$$
\begin{aligned}
& \int_{0}^{u_{\max }} \exp \left(\lambda_{1}-1+\lambda_{2} u\right) \mathrm{d} u=1 \Rightarrow \lambda_{2} \exp \left(1-\lambda_{1}\right) \\
& \quad=\exp \left(\lambda_{2} u_{\max }\right)-1
\end{aligned}
$$

Considering Eq. (15) and with $\lambda_{2} u_{\max }=G$ as entropic parameter (Chiu 1988), Eq. (14) becomes

$$
u(x, y)=\frac{u_{\max }}{G} \ln [1+(\exp (G)-1) \cdot F(u(x, y))]
$$

in which parameter $G$ is determined using the constraint expressed by Eq. (3). Equation (16) is the 2D velocity distribution in terms of $u_{\max }, G$, and 2D CDF. Substitution of Eq. (16) in Eq. (4) yields the PDF of velocity $f(u)$, which results in the uncertainty associated with the $2 \mathrm{D}$ velocity distribution or the PDF of $u$, when inserted into Eq. (1).

Using the PDF $f(u)$ defined by Eq. (4), the ratio between the mean and the maximum velocities can be derived as an exponential function of $G$ only. A constraint equation used for deriving velocity distributions, for example Eq. (3), is often referred to as the average value of velocity, for example, the average velocity in the geometric space $u_{a v}$. This average value is, however, different from $\bar{u}$ in Eq. (3), a point often overlooked in the literature. Therefore, for the cross-sectional average velocity, one must use with $A$ as the total area of the 2D domain

$$
u_{a v}=\frac{1}{A} \int_{A} \frac{u_{\max }}{G} \ln [1+(\exp (G)-1) \cdot F(u)] \mathrm{d} A
$$

Equation (17) can be solved to obtain $G$. Referring to a geometric domain in which $u_{a v}$ and $u_{\max }$ are known, one can determine first $G$ using Eq. (17) and then calculate the velocity distribution using Eq. (16), once the CDF in 2D is defined.

In practice, the method requires $u_{a v}$ and $u_{\max }$ to be measured. In particular, $u_{a v}$ is calculated from the discharge and the flow area or inferred from the ratio $u_{a v} / u_{\max }$, which was proved to be constant over time by Chiu and Said (1995), Xia (1997), and Moramarco et al. (2004). Consequently, if the stage-discharge curve is available at a certain station, only $u_{\max }$, generally simple to measure, is required to apply the $2 \mathrm{D}$ velocity model. This model invokes that the cross-section be practically invariant with time which is not always true because of water hyacinth growth, accumulation of debris, or erosion and deposition affecting the average velocity.

\subsection{The CDF in the $2 D$ case}

The CDF depends on the geometry of the domain, which must have the following properties: (1) defined between 0 and 1, (2) continuous and differentiable, and (3) its value on the borders must be 0 and have just one point in which it reaches 1 . Consider a rectangular channel in which the distribution of velocity is symmetrical with respect to the vertical axis. One can distinguish the position of coordinates, the location of $u_{\max }$ occurring on or below the water surface depending on distance $y_{0}$ of the point at the maximum velocity from the channel bed, and the size of domain $H$ (height) and $B / 2$ (half width).

It is convenient to convert this domain into a dimensionless form using the normalizing quantities $B$ and $H$ as $\psi=y / H$, 


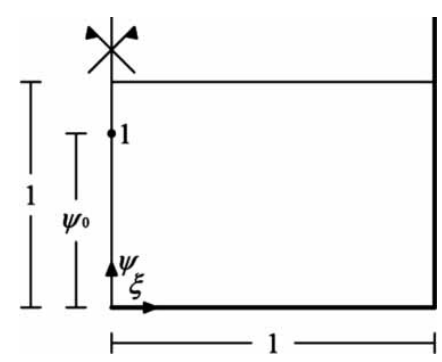

Figure 1 Symmetrical rectangular dimensionless domain

$\xi=2 x / B$, and $\psi_{0}=y_{0} / H$, in which the ratio $u / u_{\max }$ instead of $u$ is considered (Fig. 1). Using these variables and geometrical considerations (Appendix), $F(u)$ is obtained as

$$
\begin{aligned}
F(u)= & \left(1-\xi^{2}\right)^{H / B} \cdot 4 \\
& \cdot\left[\left(\frac{\psi}{2}\right)^{\ln 2 /\left(\ln 2-\ln \left(\psi_{0}\right)\right)}-\left(\frac{\psi}{2}\right)^{2 \ln 2 /\left(\ln 2-\ln \left(\psi_{0}\right)\right)}\right]
\end{aligned}
$$

Equation (18) satisfies all the aforementioned properties: it is continuous and differentiable, varies between 0 and 1 , and reaches the value 1 as $\xi=0$ and $\psi=\psi_{0}$.

The CDF $F(u)$ given by Eq. (18) has two parts: $\left(1-\xi^{2}\right)^{H / B}$ expresses the dependence on $\xi$ and the second part expresses the dependence on $\psi$. In the first part, the ratio $H / B$ appears as an exponent, meaning that as the domain is very wide and, consequently, as $H / B \rightarrow 0$, the first part of $F(u)$ tends to 1 and so $F(u)$ depends only on $\psi$. Therefore, as the domain becomes very wide, $F(u)$ must depend just on $\psi$, following also a physical intuition. Then, Eq. (18) becomes

$$
F(u)=4 \cdot\left[\left(\frac{\psi}{2}\right)^{\ln 2 /\left(\ln 2-\ln \left(\psi_{0}\right)\right)}-\left(\frac{\psi}{2}\right)^{2 \ln 2 /\left(\ln 2-\ln \left(\psi_{0}\right)\right)}\right]
$$

Equation (19) gives the 1D velocity distribution if the maximum velocity is on or below the water surface. This case was discussed by Chiu (1988), and the equations are comparable, suggesting that the 2D theory proposed here, when applied to the 1D case, represents nearly his formulation.

\subsection{Parameter $G$}

To apply the 2D velocity distribution equation given by Eq. (16), parameter $G$ must be evaluated by means of Eq. (17). Using Eq. (18) for $F(u)$, one can specify Eq. (17) as

$$
\begin{aligned}
\frac{u_{a v}}{u_{\max }}= & \int_{0}^{1} \mathrm{~d} \psi \int_{0}^{1} \frac{1}{G} \ln \left\{1+(\exp (G)-1) \cdot\left(1-\xi^{2}\right)^{H / B} \cdot 4\right. \\
& \left.\cdot\left[\left(\frac{\psi}{2}\right)^{\ln 2 /\left(\ln 2-\ln \left(\psi_{0}\right)\right)}-\left(\frac{\psi}{2}\right)^{2 \ln 2 /\left(\ln 2-\ln \left(\psi_{0}\right)\right)}\right]\right\} \mathrm{d} \xi
\end{aligned}
$$

To obtain $G$, Eq. (20) needs to be solved numerically because of its implicit form, depending on $H / B, \psi_{0}$, and $u_{a v} / u_{\max }$. The entropic parameter tends to be invariant at a channel section, whether the flow is steady or unsteady (Chiu et al. 2005). Moramarco and Singh (2010) noted that the entropic parameter is also independent of the energy or the water surface slope.

\section{Comparison with Chiu's 2D distribution}

\subsection{Chiu's distribution}

Chiu $(1987,1988,1989)$ derived a general expression for CDF of velocity by introducing equations for isovels where each curve in the physical space has a constant value of velocity. For estimating discharge during floods, Moramarco et al. (2004) developed a practical method by assuming that the $2 \mathrm{D}$ Chiu equation, written for the vertical where the maximum velocity occurs, also applies to other verticals as (Chiu 1988, 1989, Chiu and Said 1995, Greco 1999)

$$
\begin{gathered}
\frac{u}{u_{\max , i}}=\frac{1}{M_{i}} \ln \left[1+\left(\exp \left(M_{i}\right)-1\right) \frac{\psi}{\psi_{0, i}}\left(1-\frac{\psi}{\psi_{0, i}}\right)\right], \\
i=1,2, \ldots, N_{v}
\end{gathered}
$$

where $i$ is the $i$ th vertical; $\psi$, the vertical distance measured from the channel bed divided by the water depth; $\psi_{0, i}$, the value of $\psi$ with the maximum velocity; and $N_{v}$ the number of verticals sampled. Weaknesses of the above approach include requirement of too many parameters and the physical basis for using isovels. The modification of Moramarco et al. (2004) is simpler, but it still requires information on average velocity, position, and magnitude of maximum velocity for each vertical.

\subsection{Experimental measurements}

To evaluate the proposed 2D velocity distribution, experimental velocity data were collected. Table 1 presents the following characteristics: reference, number of data, channel width, water depth, maximum velocity $u_{\max }$, average velocity $u_{a v}$, relative vertical position of maximum velocity $\psi_{0}=y_{0} / H$, and value of entropy parameter $G$ from Eq. (20). Each series contains velocity observations distributed along various verticals, so that for each a $2 \mathrm{D}$ distribution of experimental velocity is obtained. Only a preliminary validation is discussed herein for the lack of more data, which is a limitation on the validation of the proposed model.

\subsection{Validation}

For each data series, velocity profiles were estimated using the proposed 2D distribution and by means of the work of Chiu (1988) and as revised by Chiu and Hsu (2006). For the data of Chiu and Hsu (2006), the observed velocity distribution, the proposed 2D distribution, and Chiu's 2D velocity distribution are shown in Fig. 2. The profiles based on the proposed velocity 
Table 1 Characteristics of velocity data

\begin{tabular}{lccccccc}
\hline Reference & $\begin{array}{c}\text { Number } \\
\text { of data }\end{array}$ & $\begin{array}{c}\text { Channel } \\
\text { width [cm }]\end{array}$ & $\begin{array}{c}\text { Water } \\
\text { depth }[\mathrm{cm}]\end{array}$ & $u_{\max }[\mathrm{cm} / \mathrm{s}]$ & $u_{a v}[\mathrm{~cm} / \mathrm{s}]$ & $\psi_{0}[-]$ & $G[-]$ \\
\hline Bortz (1989) & 29 & 121.9 & 18.3 & 78.3 & 71.6 & 0.95 & 7.032 \\
Chiu and Hsu (2006) & 29 & 61 & 18.3 & 126.3 & 90.0 & 0.62 & 0.759 \\
Guo (1990) & 13 & 10 & 2.3 & 37.9 & 29.0 & 0.72 & 0.469 \\
Run14 Guy et al. (1966) & 24 & 243.8 & 27.4 & 125.3 & 112.7 & 0.90 & 4.978 \\
Run28 Guy et al. (1966) & 25 & 243.8 & 18.3 & 131.7 & 117.7 & 0.84 & 3.716 \\
Run1 Steffler et al. (1983) & 194 & 114.3 & 14.6 & 84.4 & 75.5 & 1.00 & 7.364 \\
Run2 Steffler et al. (1983) & 307 & 114.3 & 22.5 & 54.3 & 49.0 & 1.00 & 6.353 \\
\hline
\end{tabular}

(a)

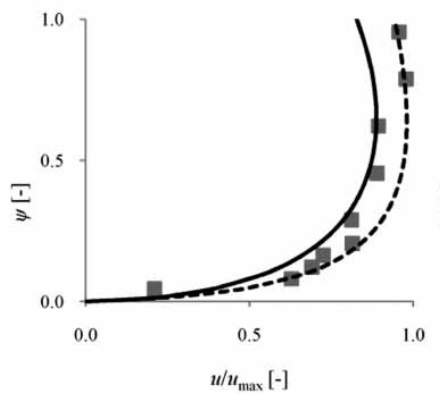

(b)

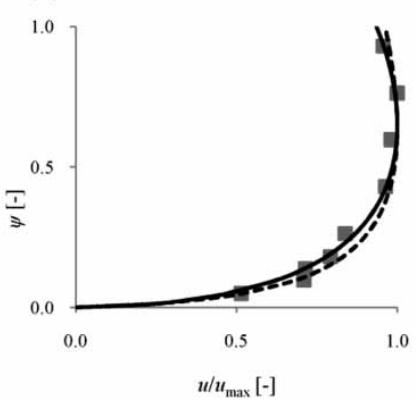

(c)

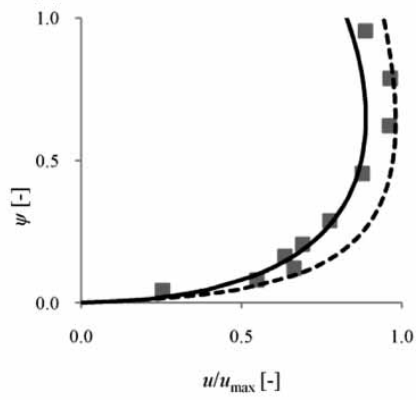

Figure 2 Comparison between experimental data and theoretical velocity profiles at section $\xi=(a)-25 \%$, (b) $0 \%$, and (c) $+25 \%$ : (-) theory, and (--) Chiu and Hsu (2006)

distribution compare better with experimental data than do the profiles of Chiu. In addition, the average $d_{a v}$ and maximum $d_{\max }$ values of these differences and three validation indices were calculated. The difference is defined as $\left|u-u^{*}\right| / u_{\max }$, in which $u^{*}$ is calculated by means of the proposed or Chiu's distribution. The validation indices of Krause et al. (2005) used were the coefficient of determination $r^{2}$, the Nash-Sutcliffe efficiency $E$, and the index of agreement $i a$.

The values of differences and indices are given in Table 2 for each series. Again, the proposed 2D distribution yields smaller differences and validation indices better than Chiu's distribution.
As stated above, Chiu's distribution needs many parameters, whereas the proposed distribution needs just the average and maximum velocities and the position of $u_{\max }$.

For the sake of brevity, the velocity profiles for other series are not presented; Fig. 3 shows the differences between the experimental and theoretical profiles inferred from the proposed 2D distribution. A similar figure for Chiu's distribution is omitted. The results given in Table 2 confirm the largest scatter of the points related to Chiu's approach with respect to Fig. 3 .

For a few points, there are large differences between experimental and theoretical values. These are related to lower velocity

Table 2 Deviations and validation indices for all series

\begin{tabular}{lcccccc}
\hline Reference & Approach & $d_{a v}[-]$ & $d_{\max }[-]$ & $r^{2}[-]$ & $E[-]$ & $i a[-]$ \\
\hline Bortz (1989) & Proposed & 0.0493 & 0.1727 & 0.8192 & 0.6609 & 0.9181 \\
& Chiu (1988) & 0.0528 & 0.1925 & 0.7668 & 0.5688 & 0.9131 \\
Chiu and Hsu (2006) & Proposed & 0.0542 & 0.1930 & 0.8936 & 0.8728 & 0.9633 \\
& Chiu (1988) & 0.0757 & 0.2801 & 0.8908 & 0.7368 & 0.9220 \\
Guo (1990) & Proposed & 0.0864 & 0.2146 & 0.8741 & 0.8399 & 0.9550 \\
& Chiu (1988) & 0.0556 & 0.3875 & 0.9594 & 0.8193 & 0.9339 \\
Run14 Guy et al. (1966) & Proposed & 0.0783 & 0.3853 & 0.8709 & 0.6519 & 0.8892 \\
& Chiu (1988) & 0.0828 & 0.3726 & 0.8719 & 0.6396 & 0.8894 \\
Run28 Guy et al. (1966) & Proposed & 0.0203 & 0.0553 & 0.9768 & 0.9469 & 0.9883 \\
& Chiu (1988) & 0.0315 & 0.0723 & 0.9711 & 0.8882 & 0.9740 \\
Run1 Steffler et al. (1983) & Proposed & 0.0555 & 0.2016 & 0.9363 & 0.8379 & 0.9683 \\
& Chiu (1988) & 0.0651 & 0.2341 & 0.9153 & 0.7780 & 0.9578 \\
Run2 Steffler et al. (1983) & Proposed & 0.0544 & 0.3691 & 0.9647 & 0.9647 & 0.9909 \\
& Chiu (1988) & 0.1063 & 0.4553 & 0.9484 & 0.5988 & 0.9318 \\
\hline
\end{tabular}




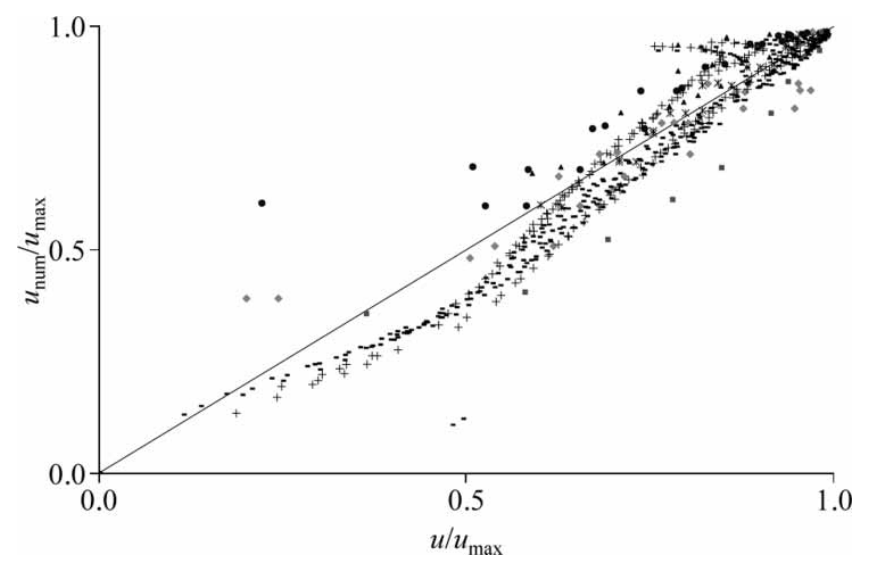

Figure 3 Comparison between experimental and theoretical data (2D proposal) with (ム) Bortz (1989), (*) Chiu and Hsu (2006), (ם) Guo (1990), (•) Run14 Guy et al. (1966), (*) Run28 Guy et al. (1966), (+) Run1 Steffler et al. (1983), and (-) Run2 Steffler et al. (1983)

areas or to boundary areas, near either the bottom or the banks. These differences are large for both Chiu's and the proposed distributions. These differences can be explained as follows: (1) Experimental data near the boundary suffer from greater uncertainty than those obtained from other areas. Thus, it is likely that these data are not as precise as expected. (2) Banks and the channel bottom significantly affect the velocity and both the proposed and Chiu's models are based on only one constraint which is based on mass conservation and do not explicitly account for boundary effects. Scatter related to points not near the boundary limits the method. Table 2 shows that the proposed 2D velocity distribution is remarkably accurate and superior to Chiu's distribution.

\section{Conclusions}

This research has developed a new 2D velocity distribution using entropy. The derivation uses a probability function defined in a 2D symmetric rectangular domain. The agreement between experimental velocity measurements and the velocity computed from the 2D distribution data is quite close. Neither parameter calibration nor isovel equation is required; only the average velocity and the maximum velocity and its position have to be known: while the first can be inferred from a stage-discharge curve or from the maximum velocity, the second has to be measured. The proposed plane velocity distribution is parsimonious and superior to existing velocity distributions.

\section{Appendix}

Consider a rectangular channel with the CDF $F[u(x, y)]$ to be searched of properties defined in Section 2.5. A CDF should be based on available experimental data and satisfy similarity in the velocity distribution, so that suitable parameters appear in the final equation depending on both $\xi$ and $\psi$. Assume that the first part depends on $\xi$ and the second part on $\psi$ only; each part must vary from 0 to 1 so that the product yields 0 on the border and 1 at $\left(0, \psi_{0}\right)$. The first part depending only on $\xi$ has to be symmetrical on the $\psi$ axis and its derivative has to be nil. The simplest equation satisfying these properties is the parabolic equation

$$
1-\xi^{2}
$$

If the rectangular domain is very wide $(H \ll B), F$ should depend not on $\xi$, but only on $\psi$, and the first part should be equal to 1 . This is satisfied by the exponent $H / B$ to obtain

$$
\left(1-\xi^{2}\right)^{H / B}
$$

The second part of $F(u)$ must depend only on $\psi$, and it must be $\psi(0)=0$, and $\psi\left(\psi_{0}\right)=1$. Starting from the simple case $\psi_{0}=1$ and using the parabolic equation, one obtains

$$
4\left[\frac{\psi}{2}-\left(\frac{\psi}{2}\right)^{2}\right]
$$

Since $\psi_{0}$ can be $<1$, Eq. (A3) exponent $a$ for each term is introduced, with $a$ as a function of $\psi_{0}$

$$
4\left[{\frac{\psi^{a}}{2}}^{a}-\left(\frac{\psi}{2}\right)^{2 a}\right]
$$

Because $a\left(\psi_{0}\right)$ must have the properties $<1$ for $\psi_{0}$ between 0 and $1,=1$ as $\psi_{0}=1$, and as $\psi=\psi_{0} \psi(1)=\psi_{0}, a\left(\psi_{0}\right)$ is determined from

$$
\left[{\frac{\psi_{0}}{2}}^{a}-\left(\frac{\psi_{0}}{2}\right)^{2 a}\right]=\frac{1}{4}
$$

Insertion of the expression for $a$ into Eq. (A4) gives

$$
4\left[\frac{\psi}{2}^{\ln (2) /\left(\ln (2)-\ln \left(\psi_{0}\right)\right)}-\left(\frac{\psi}{2}\right)^{2 \ln (2) /\left(\ln (2)-\ln \left(\psi_{0}\right)\right)}\right]
$$

The product of Eqs. (A2) and (A6) finally gives Eq. (18).

\section{Notation}

$a=$ function of $\psi_{0}[-]$

$A=$ total area of cross-section $\left[\mathrm{L}^{2}\right]$

$B \quad=$ width of cross-section [L]

$d_{a v}=$ average difference between experimental and theoretical data $[-]$

$d_{\max }=$ maximum difference between experimental and theoretical data $[-]$

$E=$ Nash-Sutcliffe efficiency validation index [-]

$f(u)=$ PDF of $u[-]$

$F(u)=\mathrm{CDF}$ of $u[-]$

$G=$ entropic parameter [-]

$H=$ height of cross-section or water depth [L] 


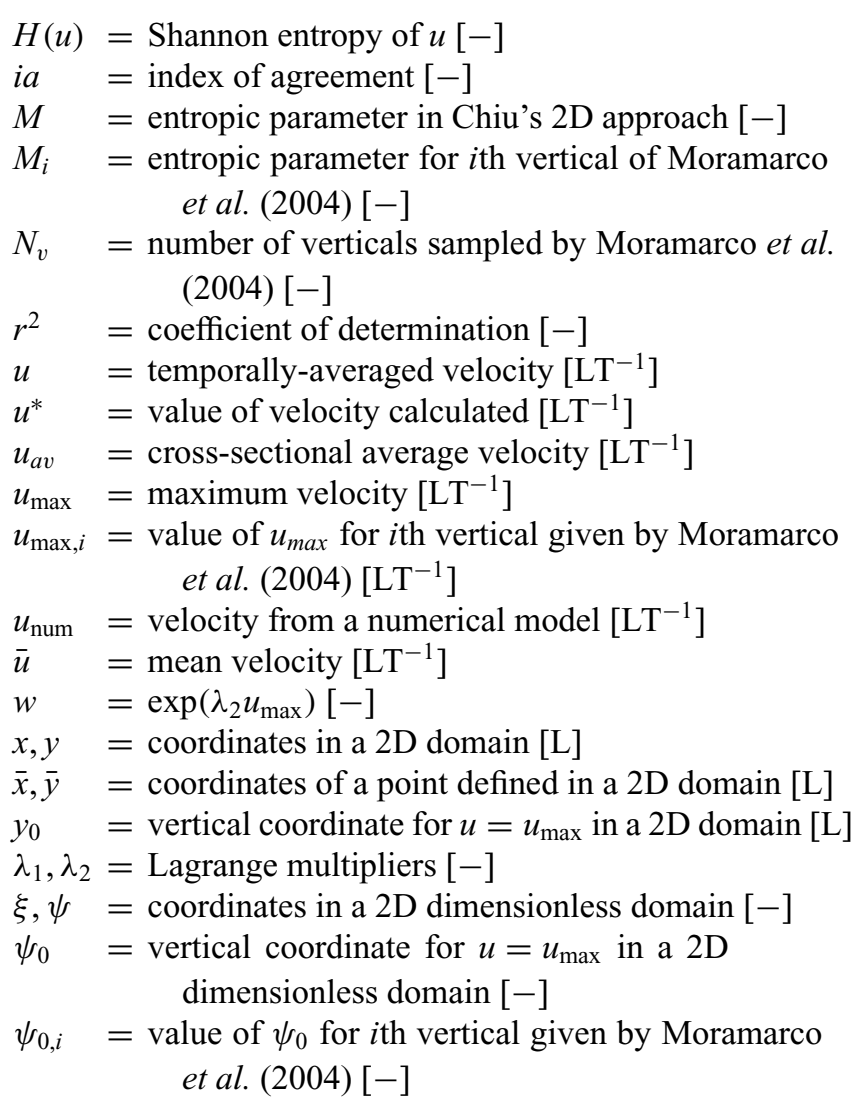

\section{References}

Barbé, D.E., Cruise, J.F., Singh, V.P. (1991). Derivation of a velocity distribution using the principle of maximum entropy. J. Hydraulic Eng. 117(10), 1389-1396.

Bortz, K.M. (1989). Parameter estimation for velocity distribution in open channel flow. M.S. thesis. Dept. Civil Engineering, University of Pittsburgh, Pittsburgh PA.

Chiu, C.L. (1987). Entropy and probability concepts in hydraulics. J. Hydraulic Eng. 113(5), 583-600.

Chiu, C.L. (1988). Entropy and 2-D velocity distribution in open channel. J. Hydraulic Eng. 114(7), 738-756.

Chiu, C.L. (1989). Velocity distribution in open channel flow. J. Hydraulic Eng. 115(5), 576-594.

Chiu, C.L., Said, C.A. (1995). Maximum and mean velocities and entropy in open-channel flow. J. Hydraulic Eng. 121(1), 26-35.

Chiu, C.L., Hsu, S.M., Tung, N.C. (2005). Efficient methods of discharge measurements in rivers and streams based on the probability concept. Hydrol. Processes 19, 3935-3946.
Chiu, C.L., Hsu, S.H. (2006). Probabilistic approach to modeling of velocity distributions in fluid flows. J. Hydrology 316, $28-42$.

Greco, M. (1999). Entropy velocity distribution in a river. Proc. IAHR Symp. River, Coastal, and Estuarine Morphodynamics Delft 2, 121-130.

Guo, Z.R. (1990). Personal communication in Chiu and Hsu, 2006. Southeast China Environmental Science Institute Yuancun, China.

Guy, H.P., Simons, D.B., Richardson, E.V. (1966). Summary of alluvial channel data from flume experiments, 19561961. G.S. Professional Paper 462-I. Geological Survey, Washington DC.

Jaynes, E.T. (1957). Information theory and statistical mechanics. Physical Rev. 106(4), 620-630.

Kirkgoz, M.S., Akoz, M.S., Oner, A.A. (2009). Numerical modeling of flow over a chute spillway. J. Hydraulic Res. 47(3), 790-797.

Krause, P., Boyle, D.P., Base, F. (2005). Comparison of different efficiency criteria for hydrological model assessment. Advances in Geosciences 5(1), 89-97.

Moramarco, T., Saltalippi, C., Singh, V.P. (2004). Estimation of mean velocity in natural channels based on Chiu's velocity distribution equation. J. Hydrologic Eng. 9(1), 42-50.

Moramarco, T., Singh, V.P. (2010). Formulation of the entropy parameter based on hydraulic and geometric characteristics of river cross sections. J. Hydrologic Eng. 15(10), 852-858.

Shannon, C.E. (1948). A mathematical theory of communication. Bell Syst. Tech. Journal 27(7), 379-423; 27(10), 623-656.

Singh, V.P., Yang, C.T., Deng, Z.Q. (2003). Downstream hydraulic geometry relations 1: Theoretical development. Water Resources Res. 39(12), 1-15.

Steffler, P.M., Rajaratnam, N., Peterson, A.W. (1983). LDA measurements of mean velocity and turbulence distribution in a smooth rectangular open channel. Dept. of Civil Eng., Univ. Alberta, Edmonton.

Tang, X., Knight, D.W. (2009). Analytical models for velocity distributions in open channel flows. J. Hydraulic Res. 47(4), 418-428.

Xia, R. (1997). Relation between mean and maximum velocities in a natural river. J. Hydraulic Eng. 123(8), 720-723.

Yang, C.T. (1972). Unit stream power and sediment transport. J. Hydraulics Div. ASCE 98(HY10), 1805-1826.

Yang, C.T. (1976). Minimum unit stream power and fluvial hydraulics. J. Hydraulics Div. ASCE 102(HY7), 919-934. 